\title{
Reflexiones acerca de salud mental y cambios en estilo de vida en adultos mayores
}

\section{Reflections on Mental Health and Lifestyle Changes in the Elderly}

"La vida es un movimiento perpetuo que cuando no puede avanzar en línea recta se transforma en movimiento circular"

Thomas Hobbes

\author{
Alejandro Klein \\ Universidad de Guanajuato, Mexico \\ Oxford Institute of Population Ageing, Reino Unido
}

\begin{abstract}
Resumen. Este trabajo busca ser un aporte al campo de la salud mental y los cambios de estilo de vida e identidad con los adultos de la tercera edad. Se tiene en cuenta el envejecimiento poblacional, pero se indica que más allá del mismo se impone revisar paradigmas estereotipados, centrados en la decrepitud, que estrechan el abordaje clínico. Es relevante la importancia de tener en cuenta que estos adultos mayores presentan nuevas formas de subjetividad que rompen con los estereotipos tradicionales. Se plantea la hipótesis de la relevancia del cuidado del encuadre en términos de garantía y confianza y la importancia de las nuevas tecnologías en un proceso de reconstrucción biográfica.
\end{abstract}

Palabras-claves: adultos mayores; identidad renovada; perspectiva clínica

\begin{abstract}
The aim of this paper is to act as a contribution to the field of the mental health and the lifestyle changes and identity shifts of the elderly. Population aging is taken into account, but this paper also encourages a review of stereotyped paradigms, focused on decrepitude, that narrow the clinical approach. The importance of bearing in mind that these elderly people present new forms of subjectivity that break with traditional stereotypes is relevant. The hypothesis of the relevance of the care of their setting, in terms of guarantees and trust, and the importance of new technologies in a process of biographical reconstruction, is addressed. Key Words: elderly, renewed identity, clinical perspective
\end{abstract}

\footnotetext{
Alejandro Klein es profesor en la Universidad de Guanajuato en México e Investigador asociado en el Oxford Institute of Population Ageing. La correspondencia sobre este artículo debe enviarse al autor al email: alejandroklein@ hotmail.com 


\section{El decisivo cambio de la sociedad de envejecimiento}

De forma inequívoca y clara los países europeos, junto con probablemente el mundo todo, están consolidando una reconfiguración poblacional en torno a un aumento cada vez mayor de la expectativa de sobrevida, con una caída en la tasa de reposición poblacional. España participa de este proceso, y según algunos indicadores, lo profundiza aún más (INE, 2010).

La esperanza de vida al nacer rondaba en España, para el año 2009, en 65 años para casi el $90 \%$ de los niños nacidos ese año y de ese porcentaje, se esperaba que alrededor de un 30\% pudiera llegar a los 90 años, lo que se acompaña además de una caída sostenida en los porcentajes de mortalidad en niños (Gènova Maleras, 2009).

Esta caída en la mortalidad no llega a compensar la imposibilidad de reposición poblacional, que se mantiene sostenidamente en España desde el año 1987. Para el año 2015 se situaba en 1,32. Uno de los índices más bajos en toda Europa. Solo Portugal es probablemente menor, con un paupérrimo índice de 1,21 (IPF, 2015).

Los adultos mayores se estimaban en España, para el año 2009, en 8 millones de personas (IMSERSO, 2009). Pero para el año 2050, España tendrá 16 millones de adultos mayores, llegando a representar prácticamente el 30\% de la población total española (INE, 2010). Habrá pues una duplicación del número de adultos mayores. Para ese mismo año se estima que los adultos mayores de alrededor de 60 o 65 años representarán $36,8 \%$ de la población total, el grupo 80 años representará el 18,2\% de la población total y significativamente, habrá casi un $12 \%$ de adultos mayores acercándose a los 100 años. (United Nations, 2008).

Esta tendencia no debería de extrañar ya que para el año 2050 se espera que el $21.8 \%$ de la población mundial será de adultos mayores, con 2008 millones de adultos mayores de 60 años y 395 millones de adultos mayores de 80 años (United Nations, 2008).

Para ese mismo año, la población de centenarios pasará de 324.000 a 4.1 millones en todo el mundo. En los países más desarrollados implicará porcentualmente un aumento de 1.119 por ciento, mientras que en los países menos desarrollados implicará un aumento de 1716 por ciento. Los porcentajes indican claramente una tendencia por la que podemos hablar de no solo de una sociedad envejecida, sino especialmente de una sociedad de centenarios (Leeson, 2009).

Quisiéramos hacer notar que esto que se presenta en cifras, representa cualitativamente el pasaje definitivo de una sociedad "adulto-céntrica" a una sociedad de "geronto-céntrica". Nunca en la historia de la Humanidad tantas personas, de forma sostenida, han ido acercándose a experiencias matusalénicas. Nunca antes la Humanidad se ha encontrado ante el desafío de reestructurar sus calles, arquitecturas, ciudades, prácticas de cuidado y salud, políticas sociales y públicas en función de que de 3 personas, 1 será un adulto mayor (Klein, 2015, 2018; Klein y Carcaño, 2017; Lorenzo Carrascosa, 2004).

Sin embargo, no pocos expertos indican que la "ventana de oportunidad" ya se ha cerrado o se cierra inexorablemente para España y Europa, en relación a la magnitud y consistencia de los cambios que se han de realizar (Leeson y Harper, 2006, 2007). El desafío está delante nuestro...

\section{El cambio de paradigma en salud mental de los adultos mayores: de la senescencia decrépita a las nuevas formas de experimentación de la tercera edad}

Los datos presentados indican además que cada vez más existirá un incremento sostenido en servicios sanitarios y de salud focalizado en adultos mayores, especialmente con personas que viven, no pocas veces, en condiciones precarias, de soledad, privación, y -probablemente- de abandono familiar (Pérez Díaz, 1998).

Se indica de esta manera que los servicios sanitarios y de salud se deben ajustar a nuevas demandas, contextos y conflictos que surgen de una población cada vez más longeva, y agreguemos además, donde existe una rencilla permanente y no resuelta sobre si es el Estado o la familia, quien se debe encargar de cuidados, atenciones y contenciones varias (Osuna et al., 2003).

Pero, el énfasis dado a lo cuantitativo, resaltando lo que falta y lo que es necesario suplir, quizás no tiene en cuenta que se necesita también un enfoque cualitativo de cambio, tan necesario como el anterior. Quizás se 
haga necesario repensar en este sentido a un autor como Erikson (1985) que funciona como un fuerte paradigma dentro de la psicología del desarrollo (Kuhn, 2005).

Erikson, como se sabe, basa su psicología evolutiva en los logros de integración entre el sujeto y la cultura. En el estadio VII o de la vejez, el autor ubica como tarea primordial el lograr una integridad yoica con un mínimo de desesperanza. Esta integridad yoica remite, al llegar al final de la vida, a un sentido de integración y plenitud, aceptando la vida que se ha vivido y por ende la muerte que se tiene por "delante". Parece ser que la idea de Erikson es que en la medida en que se valora la vida transcurrida, mejor se acepta la muerte. La persona que afronta la muerte sin miedo es lo que se llamaría sabiduría.

Sin desmerecer los desarrollos eriksonianos, da la impresión de que los mismos se nutren, de una imagen muy idealizada o ideologizada de lo que es el viejo y la vejez, en términos de "ejemplo", "moral", "integridad" y "pilar social". Probablemente hay personas que "sabiamente" acepten su muerte, para lo cual no necesariamente hay que ser viejo, si tenemos en cuenta que cualquiera puede morir en cualquier momento, pero cabe preguntarse si las ideas de "vida" y "muerte" que maneja el autor no han sufrido un cambio radical en términos sociales y culturales en lo que concierne al adulto mayor.

Erikson no pudo prever las transformaciones que ocurrieron en relación a los adultos mayores en las últimas décadas. Transformaciones que van más allá de la transición demográfica y resignifican la misma, en términos por las cuales el adulto mayor ya no ve delante de sí una muerte inminente, sino una muerte desplazada. Este desplazamiento indefinido lo coloca en una nueva búsqueda de oportunidades, de proyectos, de aspiraciones y anticipaciones jubilosas de vivir. Al adulto mayor de hoy parece que no le interesa ser más "sabio", sino experimentar nuevas e inéditas versiones de sí (Bengtson, 2004; Eisenberg, 1988; Fisher, 1983; Harper, 2004; Neugarten y Neugarten, 1986).

Es importante que las consideraciones teóricas con que se consolidan los dispositivos de salud mental dirigidos a adultos mayores, tengan en cuenta que hay actualmente nuevas versiones y nuevos conflictos del adulto mayor (Neugarten y Weinstein, 1961; Wilcoxon, 1987). Los procesos de psicoterapia van tomando en cuenta paulatinamente esta realidad, generando reflexiones pertinentes en tal sentido, aunque quizás falté ahondar un diálogo interdisciplinario más fluido entre la psicología, la demografía, el trabajo social, entre otros (Aducci, 2004; Ballesteros Jiménez, 2004; Neugarten, 1982; Salvarezza, 1988).

De otra manera, cabe el peligro de que se enfoque la atención a los adultos mayores exclusivamente desde una perspectiva de análisis de la senescencia, la posible demencia, el deterioro, la pérdida y los déficits o los duelos, que si bien se constatan y son relevantes, se acompañan otras veces de conductas de resiliencia y empoderamiento (Carstensen, 1990; Coderch, 1990).

Cabe indicar que las dificultades mentales, sociales, sanitarias, laborales, económicas, familiares, entre otras, en que se encuentra el adulto mayor, no solo reflejan un déficit crónico de políticas sociales, sino además la reiteración de un estereotipo de una imagen de anciano desvalido y desamparado (Klein, 2016b). En este estereotipo, el adulto mayor es calificado de "viejo" y "anciano", buscando destacar su vulnerabilidad y decrepitud y se lo supone especialmente enfrentado a la muerte, la soledad y el desamparo.

Se asume por ende que todo sujeto adulto mayor pasa por lo mismo, como si no hubiera situaciones de envejecimiento diferentes y cambiantes. Es alguien que ya está a punto de morir, por no decir que agoniza existencialmente, lleno de recuerdos, duelos y nostalgias, enfrentado a una serie sucesiva de pérdidas que lo atormentan o lo deprimen. El proceso de envejecimiento aparece inevitablemente como un proceso de déficit crónico e irreversible (Klein, 2010).

Al ser una persona tan vulnerable y carente, las políticas públicas, mentales o sociales van a ser concebidas básicamente para cubrir este déficit. Comparado con una adultez capaz de autonomía, vigor, y redituable productivamente, el anciano aparece caracterizado por ser improductivo e inútil (Huenchuan, 2004).

Incapaz de autonomía, tomar decisiones y auto-sustentarse, está sujeto a un proceso de regresión infantilizante. Pierde no solo su condición física y mental, sino también su dignidad social y estética, lo que lo transforma en un ser incapaz. Su destino no puede ser entonces sino el estar solo, en la calle o en un asilo público en situaciones de ruina, soledad y abandono (Garatachea, 2007; Katz, 2000). 
Una versión renovada de la psicoterapia con la tercera edad debe necesariamente tener en cuenta la nueva versión que la tercera edad está haciendo de sí misma

Por el contrario, paulatinamente se va afirmando otra forma de identidad de los adultos mayores. Se trata de una perspectiva donde lo que cuenta es la vida, la continuación de vida y no la inminencia de la muerte (Klein, 2016a). Esta búsqueda y reclamo de plenitud de vida, reformula audazmente ideas estereotipadas, indicando que ya no hay viejos ni ancianos, sino "adultos mayores", "tercera edad" u otros calificativos, que fortalecen la imagen de fuerza, plenitud, iniciativa y capacidad de resolver conflictos (Boerner y Joop, 2007).

El paradigma de plenitud de vida lleva al compromiso con la calidad de vida, desde la cual se impone un sujeto adulto mayor lleno de potencialidades más allá o en contra, del proceso de envejecimiento. Ya no se trata del déficit y de la pérdida, sino de la oportunidad del proyecto. Mientras que desde el paradigma de la vulnerabilidad el anciano está exiliado de lo social, desde la calidad de vida se lo reubica como "adulto mayor" o "tercera edad" en el centro de la esfera social (Ham Chande, 1999).

Se trata de una persona productiva, con plena capacidad de sus fuerzas mentales, emocionales y corporales. Se asocia al sentido de esperanza, optimismo y vitalidad legitimado socialmente. Aquí interesarán los procesos por los cuales puede llegar a ser y mantenerse autónomo (Bryant et al., 2001). Ya no es resignarse a la soledad, sino que se acepta y se disfruta la compañía del grupo (Arias, 2013).

Investigaciones recientes dan muestra de acciones de este colectivo que conlleva a procesos autonómos, rompiendo con los modelos estereotipados sobre la tercera edad que insisten en procesos deficitarios y que no incluyen el papel protagónico y novedoso de los adultos mayores en sus redes sociales, sus familias y su cotidianeidad. La perspectiva de la alta experimentación alternativa propone tomar en cuenta estos procesos que en realidad ya tienen su propia historia y que sin embargo hasta el momento han sido invisivilizados, por paradigmas dominantes que terminan por ser empobrecedores en su análisis (Klein-Carcaño, 2018).

Especialmente, surge la necesidad de tener en cuenta nuevos escenarios alternativos donde los grupos de tercera edad además de combatir y sufrir procesos económicos, políticos y sociales que les han sido tradicionalmente adversos y los han vulnerabilizado, se encuentran en una posición que los perfila hacia formas inéditas y novedosas de articulación económica, cultural y social (Ham Chande, 1999).

Las políticas sociales, de esta manera, ya no se pueden enfocar solo en el desvalimiento o la vulnerabilidad, sino además en el fortalecimiento de este progresivo empoderamiento. No puede dejar de notarse que ante esta nueva concepción del adulto mayor, las políticas sociales todavía deben hacer un profundo ajuste (Klein, 2016b).

Las políticas de salud mental y las prácticas psicoterapéuticas pueden recoger este desafío y renovarse junto a esta renovación del adulto mayor, para dar cuenta de una demanda, que todo lo indica, será cada vez mayor y relevante. Psicólogos, terapeutas, psicoanalistas, técnicas y profesionales de la salud mental, tienen ante sí un enorme desafío. En lo que sigue intentaremos brindar algunas reflexiones acerca de este modelo renovado que se propone.

\section{Las inquietudes que transmiten estos adultos mayores}

Los pacientes adultos mayores tenidos en cuenta presentan unanimidad en que de ninguna manera aceptan o desean ser tratados como "viejos". Alrededor del término asocian connotaciones negativas o peyorativas de las que reniegan profundamente.

Cuando revisan su biografía o su infancia, hacen especial hincapié en que ellos son abuelos profundamente diferentes de los abuelos que les tocaron. Insisten en su capacidad de diálogo y de encuentro con sus nietos, así como remarcan cómo intentan mantener una vida social activa y enriquecedora. Aquellos que aún pueden seguir trabajando, lo siguen haciendo con alegría y compromiso. No ven a la jubilación ni como algo imperativo ni como punto final de un ciclo de vida, comprometidos con diferentes actividades: gimnasias, dietas, 
práctica sexual, nuevas búsquedas emocionales, concreción de proyectos educativos alternativos (Barros y Castro, 2002; Klein, 2010).

En ese sentido, una parte del proceso terapéutico daría la impresión de que pasa por una resignificación y extensión de su biografía. Transmiten algunas veces la queja de que sus hijos no quieren o no pueden comprender, su necesidad renovada de autonomía, emprendimientos o aventuras. En un caso se planifica un viaje, en otro volver a estudiar, y en otro aparece deseo de concretar nuevos proyectos de pareja.

Un motivo por el que consultan se desarrolla en torno al duelo por la muerte progresiva de amigos y hermanos que van falleciendo y la pregunta sobre: “¿por qué soy yo el que sobrevivo?”. Situación a la que se unen vivencias de soledad y de culpa. Otro motivo se relaciona a las patologías emergentes de una larga vida conyugal: aburrimiento, agresividad, indiferencia hacia el otro. Se trata quizás de una variante de la psicopatología de la vida cotidiana y un efecto inesperado del alargamiento de expectativa de vida: las parejas deben rearmar estructuras de convivencia y cotidianeidad luego de 40, 50 o 60 años de estar juntas (Berenstein y Puget, 1988).

Otro motivo de consulta se relaciona al conflicto entre el deseo de seguir desarrollando emprendimientos personales y la demanda familiar del cuidado o atención de nietos. Como ya señalamos no siempre estos abuelos quieren ser abuelos como los que tuvieron. Capítulo aparte merece el duelo por el cuerpo de la vejez, frente al cual advierten, no necesariamente un cuerpo "decrépito", sino un cuerpo que debe ser administrado de otra manera, lo que genera preocupación y ansiedad, pero también curiosidad.

\section{Conflictos familiares. Incomprensión}

La familia no siempre entiende, y no pocas veces, no tolera, que este adulto mayor no sea un viejo en el sentido tradicional del término, y menos aún, que no sea un viejo decrépito. Parece más preparada para roles estereotipados, que para aceptar que el adulto mayor busque una nueva pareja una vez viudo, para que emprenda una nueva educación o para que salga de viaje con su grupo de amigos.

La familia asimismo resiste mucho que estos adultos mayores no deben ser necesariamente cuidados. Los cuadros de depresión que a veces advertimos se relacionan a adultos mayores que se quejan de no ser comprendidos, y menos aún tolerados. No quieren cuidar ni ser cuidados. No quieren rechazar ni ser rechazados, reivindicando la regulación de su autoestima, la capacidad de tolerar la ansiedad de su autonomía o la vitalidad de su deseo (Berenstein, 1981).

No pocas veces, es positivo hacer entrevistas familiares, siempre con el paciente presente, para evitar situaciones regresivantes o infantilizantes. Estas entrevistas se verifican al comienzo, durante y al final del proceso.

Un capítulo aparte lo merece el vínculo con sus nietos, especialmente si estos son adolescentes. Refieren al mismo como especialmente valorado, construido desde situaciones de igualdad, intercambio y mutuo respeto. Insisten en que con sus nietos desean ensayar nuevas formas y cualidades de vida. Confían en ellos y valorizan la confianza que ellos les puedan transmitir (Vidal y Menzinger, 2005).

\section{La experiencia de seguridad: una experiencia que se va perdiendo en la vida cotidiana}

En un primer momento el adulto mayor llega con disminución de autonomía e inseguridad (Fonagy, 2000), buscando re-establecer un marco de confianza, seguridad y reaseguramiento durante el proceso (Kächele y Thomä, 1989). El paciente adulto mayor observamos que revisa aspectos de su biografía en relación al pasado, pero también en relación a proyectos de presente y futuro. La capacidad de calma, discriminación y manejo de la angustia es relevante para tomar decisiones acertadas (Ortiz, 2002).

Transferencialmente, parece que se perfila la necesidad para estos adultos mayores de contar con un terapeuta especialmente disponible, capaz de cuidar y proteger y de poder pensar junto a ellos (Bleichmar, 1997). Y sentir contratransferencialmente que se confía también en su posibilidad de cambio y que no se los enjuicia por decisiones que pueden parecer impropias para una persona de la tercera edad. 
Postulamos la hipótesis de que el proceso terapéutico apunta a la consolidación de una zona de la mente, en tanto surge como marco de integración para pensar (Bollas, 1991, 1993). Esta zona de la mente se asocia a una capacidad de mentalizar o función reflexiva, que denota la comprensión de la conducta de uno mismo en términos de estados mentales (Fonagy, 1999), alejando los fantasmas de la locura, el desconcierto o lo desorganizado.

La función reflexiva transmite que las cosas pueden ser pensadas y solucionadas. Y que esas cosas pensadas y solucionadas pueden serlo por la mente del paciente. Así se deposita confianza en uno mismo, a la vez que se transmite un contexto terapéutico estable, e imprescindible para que las cosas adquieran un sentido y una posible interpretación, con el tiempo adecuado de elaboración (Fonagy, 2000) .

Para estos adultos mayores que transmiten la necesidad de ser escuchados y tolerados en su capacidad de cambio, parece fundamental ofrecerles la posibilidad de instaurar una "escena transformacional", capaz de investimento del cambio (Klein, 2006) y de tolerar "inquietud" (Giovacchini, 1989), sin que la misma implique derrumbe psíquico.

\section{La experiencia de seguridad: una situación que se va acentuando}

De acuerdo al material preliminar tenido en cuenta, estos adultos mayores llegan a consulta con sentimientos depresivos y confusos, matizados con relatos de maltrato familiar, quejas por la vida de pareja o duelos por la muerte de hermanos y amigos.

En este sentido ya no encuentran con quien hablar y suponen que el terapeuta les puede ayudar retomando un diálogo que han perdido con aquellos. Aunque puede haber sensación de soledad, la misma no se corresponde con aislamiento: mantienen activamente redes sociales, que en algunos casos hasta son de tipo virtual.

Cabe mencionar en este sentido, como auxiliar importante de estos procesos, el uso de las nuevas tecnologías como skype o whatsapp en casos de convalecencia o enfermedad. Esta situación se acentúa en épocas de frío donde para el adulto mayor se hace difícil trasladarse. O donde siente que no le conviene salir de su casa. Son situaciones donde las tecnologías de comunicación ayudan fundamentalmente a que el encuadre siga preservando su calma y seguridad.

El uso de las tecnologías de información como terciarios simbólicos, hacen posible sostener el proceso terapéutico desde una "prótesis" de investimento de la ausencia. Por otro lado se observa que la continuidad de sesiones genera una sensación de permanencia y estabilidad. Muchas veces la sesión debe ser la garantía de la próxima sesión, desde una continuidad que garantice organización espacio-temporal de sus vidas y resuelva el par ausencia-presencia (Winnicott, 1972).

Desde esta situación, parecería que junto a intervenciones que ayuden a pensar, se necesita además instaurar un vínculo que sostenga confianza y experiencias amparadoras (Martin Montoliu, 2008).

\section{Joaquín: el conflicto con los hijos usurpadores}

Se reseña a continuación un caso abordado hace ya un tiempo considerable. Se han modificado datos significativos a efectos de preservar la identidad de la persona.

Joaquín tiene 68 años de edad. Pertenece a la clase media alta, empresario. No aparenta su edad, se viste y se presenta de forma jovial e informal: "No quiero parecer viejo. No me siento viejo. Me gusta ser abuelo, pero no un hombre débil o que necesita ayuda. No necesito que me ayuden en mi vida, sí que me respeten y consideren".

Destaca dos grandes problemas que le preocupan. Es dueño de una fábrica de la cual no quiere jubilarse, a lo que sus hijos, también propietarios de la misma, le insisten en que es hora de su retiro. De esta manera, no pocas reuniones familiares han terminado en agrias discusiones y recriminaciones. Los hijos le reprochan que obstaculiza decisiones que ellos toman y que decisiones de Joaquín entorpecen, no pocas veces, la marcha del negocio: "la empresa la hice yo, de la nada, y ahora estos desagradecidos me quieren echar, hacerme un tonto, un inútil". 
Se observa de esta manera que probablemente además de la amenaza de destitución, lo que afecta especialmente al paciente es la posibilidad de ser olvidado por sus hijos y descendientes. Ansiedad frente al olvido que explica tal vez el aferramiento obstinado del paciente sobre el o los destinos de su legado, como una forma de anular el posible olvido y restaurar permanentemente la memoria sobre él y lo que ha podido lograr en la vida.

Por otro lado, está profundamente enojado con su mujer, la que siente que no lo apoya, desde una actitud de complicidad de los hijos: "es a mí que tiene que darme la razón, está vieja, a veces es insoportable, no la entiendo, los domingos que no trabajo nos aburrimos, ella siempre con su televisión..."

Aparece como fantasía de enfermedad su "debilidad" frente a hijos a los que ubica persecutoriamente como capaces de parricidio y en los que deposita, por otro lado, su propio deseo no asumido, de salir del negocio familiar y comenzar una nueva vida, lo que le genera agobio y confusión (Kancyper, 2013).

A lo largo del proceso Joaquín va pudiendo entender que más allá de aspectos agresivos, sus hijos están intentando ayudarlo, no atacarlo. Asimismo, va aceptando en él la agresividad hacia los mismos y por ende, hacia su empresa, comprendiendo que ha tomado medidas que han boicoteado la misma. Progresivamente, pero con dificultades, se va reconciliando con los mismos, aceptando una especie de negociación, por la cual acepta un retiro a medias, por el cual sigue presente en la empresa, pero aprendiendo a confiar en la capacidad de sus hijos para tomar decisiones acertadas. Lo que a su vez calma su culpa en relación a los proyectos de vida que le van surgiendo como importantes, entre ellos viajar y recorrer el mundo.

Su rivalidad con los hijos disminuye, y el sentido de confianza ayuda a amortiguar sensaciones paranoicas de conspiración y ataque. En determinado momento le hace un planteo a su esposa de comenzar a hacer viajes juntos. En un primer momento él acapara la planificación de los mismos, comprendiendo luego que de vuelta vuelve a colocar a su esposa en ese lugar de "vieja" que él le reclamaba.

Durante uno de esos viajes plantea mantener las sesiones por medio de skype, debido a su necesidad de no hacer cortes bruscos y de sentir la confianza del terapeuta en sus nuevos emprendimientos. En determinado momento y al final de un largo viaje, Joaquín decide dar por terminado también su "viaje" terapéutico, lo que comenta de buen humor al concluir la última sesión.

\section{Conclusión}

Quisiéramos indicar como, junto a procesos terapéuticos que tienen que dar cuenta de procesos deficitarios de senescencias, duelos relevantes y deterioros cognitivos, es probable que el campo terapéutico, clínico y de la salud mental se deba ir proveyendo de dispositivos que den cuenta además, de demandas en relación a profundas resignificaciones de biografía, nuevas búsquedas emocionales y vinculares, y la presencia de la ambivalencia en torno al par autonomía- dependencia del grupo familiar.

El caso clínico presentado indica que el proceso de envejecimiento es indisociable asimismo de una delegación de poder, y su contrario: como un aferramiento al mismo ante el temor de ser olvidado o "desterrado" de la memoria filial y generacional. Incluso analizando la transferencia de poder en tanto se expresa como transferencia económica-numérica, la resistencia a este proceso podría ubicarse como un intento de preservación del cuerpo adulto que se pierde, recordando la reflexión lacaniana en torno a que el cuerpo se deja escribir (significar) por algo que es del orden del número (Lacan, 1984). En su extremo podría llevar a situaciones de estados de somnolencia hipnoide, con rasgos paranoides donde el paciente se supone a merced de las cuentas realizadas por un especulador (en el caso presentado: los hijos, la descendencia) (Maldavsky, 1992).

Se propone como estrategia terapéutica la hipótesis de consolidación de lo mental como estructura de mediación fundamental entre el aparato psíquico y el mundo externo, entre la biografía del pasado y la que se recomienza a elaborar y entre ausencia y presencia. Se trata de consolidar una estrategia de funcionamiento mental caracterizado por la consolidación de un self genuino, calmo e íntegro (Bollas, 1991, 1993). De alguna manera se relaciona a la experimentación subjetiva que estos adultos mayores plantean en términos de renovación, alta experimentación y empoderamiento.

Parece importante señalar que es positivo un ritmo continuado y asegurador en los abordajes emprendidos. De ahí nuevamente la relevancia del uso de las nuevas tecnologías para intentar volver a reconstruir y consoli- 
dar la espera y la presencia confiable, dentro de los ritmos del proceso terapéutico (Winnicot, 1972).

Es importante recalcar que el material clínico referido permite inferir otros rasgos fundamentales en el trabajo con adultos mayores referidos a la necesidad de asegurar procesos psíquicos y vinculares dirigidos a la producción de un objeto sucesor, con una pulsión por ende dirigida a la transmisión, y la angustia subsiguiente de intrascendencia (Maldavsky, 2000), en caso de que estos procesos no prosperen o se anulen. La angustia de los adultos mayores efectivamente muchas veces ronda en torno a la cuestión del legado, cómo será preservado o destruido o denigrado, lo que se relaciona a ansiedades que pueden remitir a regresiones libidinales. En este sentido podríamos retomar la hipótesis de libido intrasomática (Maldavsky, 2000), situada antes que la libido oral y cuya presencia asegura la necesidad de erigir una coraza que pueda tramitar primariamente los estímulos, los que pueden tornarse, en caso contrario, excesivos y por lo tanto, potencialmente tóxicos o traumáticos. Podríamos entonces suponer que la vejez también requiere de una reedición de libido intrasomática para regular de forma no-traumática procesos y estímulos en torno a objetos duelados, pérdidas y desarraigos que pueden a llegar a ser invasivamente traumáticos y dañinos para un psiquismo en proceso de alta resignificación. En este sentido podríamos postular, como una hipótesis que requiere mayor profundidad, la necesidad de una reorganización del aparato psíquico en el sujeto envejecido en términos de aislamiento transitorio, filtro y barrera de protección.

Notamos así, que tanto desde el campo de la subjetividad, como desde la psicopatología, están emergiendo procesos nuevos e inesperados, producto de la expectativa de sobrevida, tanto como en relación a malestares, agresividades, dificultad de reacomodación en parejas que llevan más de 50 años de coexistencia. Existe allí un campo de trabajo que ha de llevarnos a reflexionar y sobre el que se hace necesario investigar.

Cada vez más se incrementará la demanda de pacientes de la tercera edad y los abordajes clínicos deben dar respuestas satisfactorias que generen beneficios en términos de salud mental, bienestar psicológico y logros personales. La preocupación de este trabajo es hacer un aporte al debate de la situación.

\section{Referencias}

Aducci, E. (2004). Adultos mayores. Su psicoanálisis hoy. Editorial Letra Viva.

Arias, C. (2013). Aportes del apoyo social en el delineamiento de políticas públicas para las

personas mayores. En V. Montes de Oca (Ed.). La agenda del Envejecimiento y las Políticas Públicas Hoy (pp. 245-263). Instituto de Investigaciones Sociales.

Ballesteros Jiménez, S. (2004). Gerontología, un saber multidisciplinar. Ed. Universitas.

Barros, R. y Castro, A. (2002). Terceira Idade: o discurso dos experts e a produção do novo velho. Estudos Interdisciplinares sobre o Envelhecimento, 4, 113-124.

Bengtson, V. L. (2004). Beyond the nuclear family: The increasing importance of multigenerational relationships en American society. Journal of Marriage and the Family, 63(1), 1-16. https://doi.org/10.1111/j.17413737.2001.00001.x

Berenstein, I. (1981). Psicoanálisis de la Estructura Familiar. Del destino a la significación. Paidós.

Berenstein, I. y Puget, J. (1988) Psicoanálisis de la Pareja Matrimonial. Paidós.

Bleichmar, H. (1997). Avances en Psicoterapia Psicoanalítica. Hacia una técnica de intervenciones específicas. Paidós.

Boerner, K. y Joop, D. (2007). Improvement/Maintenance and Reorientation as Central Features of Coping with Major Life Change and Loss: Contributions of Three Life-Span Theories. Human Development, 50(4), 171-195. https://doi.org/10.1159/000103358

Bollas, C. (1991). La sombra del objeto. Psicoanálisis de lo sabido no pensado. Amorrortu.

Bollas, C. (1993). Fuerzas de destino. Psicoanálisis e idioma humano. Amorrortu.

Bryant, L. L., Corbett. K. K. y Kutner, J. S. (2001). In their Own Words: A Model of Healthy Aging. Social Science and Medicine, 53(7), 927-941. https://doi.org/10.1016/S0277-9536(00)00392-0

Carstensen, L. (1990). Cambios relacionados con la edad en la actividad social. En L. L. Carstensen y B. A. Edelstein (Eds). Gerontología clínica. Intervención psicológica y social (pp. 58-73). Martínez Roca. 
Coderch, J. (1990). La psicoterapia psicoanalítica en los pacientes de edad avanzada. Herder.

Eisenberg, G. (1988). Grandchildren's perspectives on relationships with grandparents: the influence of gender across generations. Sex Roles, 19, 205-217. https://doi.org/10.1007/BF00290155

Erikson, E. (1985). El ciclo vital completado. Paidós.

Fisher, L.R. (1983). Transition to grand motherhood. International. Journal of Aging and Human Development, 16(1), 67-78. https://doi.org/10.2190/GU3R-506F-2UMW-6L8R

Fonagy, P. (1999). Persistencias transgeneracionales del apego: una nueva teoría. Aperturas Psicoanalíticas, (3). https://aperturas.org/articulo.php?articulo=0000086

Fonagy, P. (2000). Apegos patológicos y acción terapéutica. Aperturas Psicoanalíticas, (4). https://aperturas. org/articulo.php?articulo=104

Garatachea, N. (2007). Actividad física y envejecimiento. Editorial Wanceulen.

Gènova Maleras, R. (2009). Presente y futuro de la longevidad de la población española en el contexto de los países de alta esperanza de vida. En L. López Trigal, A. Abellán García y D. Godenau (Eds.). Despoblación, envejecimiento y territorio: un análisis sobre la población española (pp. 333-346). Universidad de León.

Giovacchini, G. (1989). Adolescentes borderline. Nueva Visión.

Ham Chande, R. (1999). Conceptos y significados del envejecimiento en las políticas de

población. En Consejo Nacional de Població - Conapo (Ed.) Envejecimiento demográfico de México: retos y perspectivas (pp. 41-54). Conapo.

Harper, S. (2004). Families in Ageing Societies. A Multi-Disciplinary Approach. Oxford University Press. https://doi.org/10.1093/0199251169.001.0001

Huenchuan, S. (2004). Marco legal y de políticas en favor de las personas mayores en América Latina. Centro Latinoamericano y Caribeño de Demografía-División de Población de la Cepal.

IMSERSO (2009). Las personas mayores en España. Datos estadísticos estatales y por

Comunidades Autónomas. Informe 2008/tomo I. Recuperado de https://www.imserso.es/InterPresent2/groups/ imserso/documents/binario/infppmm2008v1.pdf

INE (Instituto Nacional de Estadística). (2010). Proyección de población a largo plazo. 2009-2049. Recuperado de http://www.ine.es/jaxi/menu.do?type=pcaxis\&path=/t20/p270/2009-2049\&file=pcaxis

IPF (Instituto de Política Familiar) (2015). Demografía y natalidad en España 2015. Recuperado de http:// civica.com.es/informes/informe-sobre-demografia-y-natalidad-en-espana-2015-del-instituto-de-politica-familiar-ipf/

Kächele, H. y Thomä, H. (1989). Teoría y práctica del psicoanálisis. Herder.

Kancyper, L. (2013). Volviendo a pensar con Willy y Madeleine Baranger: nuevos desarrollos. Lumen Argentina.

Katz, S. (2000). Busy Bodies: Activity, Aging, and the Management of Everyday Life. Journal of Aging Studies, 14(2), 135-152. https://doi.org/10.1016/S0890-4065(00)80008-0

Klein, A. (2006). Adolescentes sin Adolescencia: Reflexiones en torno a la construcción de subjetividad adolescente bajo el contexto neoliberal. Psicolibros Universitario.

Klein, A. (2010). Nuevas formas de familias, paternidades y relaciones familiares comomodelo de intersecciones intergeneracionales. Ageing Horizons, 9, 74-81.

Klein, A. (2015). Del Anciano al Adulto mayor. Procesos psicosociales, de salud mental, familiares y generacionales. Plaza y Valdez Editores.

Klein, A. (2016a). De la ancianidad al adulto postmayor. Desacatos, 50, 156-169. https://doi. org/10.29340/50.1547

Klein, A. (2016b). Las políticas de salud mental en México para niños, adultos y ancianos: mucho por hacer, mucho más por reflexionar. O social em questão, 19(36), 89-104.

Klein, A. (2018). La vejez problematizada. Imaginarios sociales que toleran lo que otrora era intolerable. Desacatos, 57, 120-135. https://doi.org/10.29340/57.1954

Klein, A. y Carcaño, E. (2017). La vejez problematizada. Imaginarios sociales que toleran lo que otrora era intolerable. Revista de Ciências Humanas, 51(2), 477-493. https://doi.org/10.5007/2178-4582.2017v51n2p477

Kuhn, Th. S. (2005). La estructura de las revoluciones científicas. Fondo de Cultura Económica de España.

Lacan, J. (1984). Seminario 11, Los cuatro conceptos fundamentales del psicoanálisis. Paidos. 
Leeson, G. (2009). Demography, Politics and Policy in Europe. En P. Ludlow (Ed.). Setting EU Priorities 2009 (pp. 102-124). The European Strategy Forum.

Leeson, G. y Harper, S. (2006). Attitudes to Ageing and Later Life. Global Ageing Survey. The Oxford Institute of Population Ageing.

Leeson, G. y Harper, S. (2007). Ageing and Later Life. United Kingdom and Europe. Global Ageing Survey. Research Report 107. The Oxford Institute of Population Ageing.

Lorenzo Carrascosa, L. (2004). Consecuencias del envejecimiento de la población: el futuro de las pensiones. Recuperado de https://www.ine.es/daco/daco42/sociales/infosoc envej.pdf

Maldavsky, D. (2000). Lenguaje, pulsiones, defensas. Nueva Visión.

Maldavsky, D. (1992). Teoría y clínica de los procesos tóxicos. Amorrortu.

Martin Montoliu, J. (2008) Psicoterapia en la edad tardía. Clínica y Salud, 19(1), 101-120.

Neugarten, B. y Neugarten, D (1986). Age in the aging society. En A. Piíer y L. Bronte, (Coords.). Our aging society: Promise and paradox (pp. 123-132). W.W. Norton.

Neugarten, B. y Weinstein, K. (1961). The chanching american grandparents. Journal of Marriage and Family, 26(2), 199-204. https://doi.org/10.2307/349727

Neugarten, B. (1982). Psychological aspects of aging and illness. Clinical Issues in Geriatric Psychiatry, 25(2), 123-131. https://doi.org/10.1016/S0033-3182(84)73081-7

Ortiz, E. (2002). Las palabras para decirlo. Un enfoque intersubjetivo de la comunicación en psicoterapia. Aperturas Psicoanalíticas, (12). http://www.aperturas.org/articulos.php?id=0000220\&a=Las-palabras-para-decirlo-un-enfoque-intersubjetivo-de-la-comunicacion-en-psicoterapia

Osuna, M. J., Villar, F. y Triadó, C. (2003) Rutinas cotidianas en la vejez: patrones de actividad e influencia del sexo y la edad. Revista multidisciplinar de gerontología, 13(1), 29-36.

Pérez Díaz, J. (1998). La demografía y el envejecimiento de las poblaciones. En A. S. Staab y L. C. Hodges (Eds.). Enfermería Gerontológica (pp. 451-463). McGraw Hill.

Salvarezza L. (1988). Psicogeriatria. Teoría y clínica. Paidós.

United Nations (2008). World Population prospects,2008 revision. http://www.un.org/esa/population/publications/wpp2008/wpp2008 highlights.pdf

Vidal, S. y Menzinger, J. (2005). New Technologies in the Communication between Grandparents and Grandchildren in Spain. Generations Review, 15(4), 26-32.

Wilcoxon, S. (1987). Grandparents and grandchildren: an often neglected relationship between significant others. Journey of Counseling and Development, 65, 289-290. https://doi.org/10.1002/j.1556-6676.1987. tb01287.x

Winnicott, D. (1972). Realidad y Juego. Gedisa. 